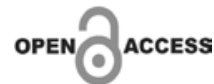

Correspondence addresses:

Dr. Gilson Soares Feitosa

gilson-feitosa@uol.com.br

Received: April 9, 2021

Revised: May 10, 2021

Accepted: June 11, 2021

Published: June 30, 2021

Data Availability Statement: All relevant data are within the paper and its Supporting Information files.

Funding: This work was the result of authors' initiative. There was no support of research or publication funds.

Competing interests: The authors have declared that no competing interests exist.

Copyright

(C) 2021 by Santa Casa de Misericórdia da Bahia.

All rights reserved.

ISSN: 2526-5563

DOI: 10.35753

\section{Therapeutic versus Prophylactic Anticoagulation for Patients Admitted to Hospital with COVID-19 and Elevated D-dimer Concentration (ACTION): An Open-Label, Multicentre, Randomised, Controlled Trial}

\author{
Anticoagulação Terapêutica versus Profilática para Pacientes \\ Internados no Hospital com COVID-19 e Concentração \\ Elevada de D-dímero (ACTION): Estudo Aberto, Multicêntrico, \\ Randomizado e Controlado
}

Gilson Soares Feitosa-Filho' ${ }^{1}$, Gabriella S. Sodré ${ }^{1}$, Juliane Penalva C. Serra ${ }^{1}$, Rhanniel Theodorus-Villar ${ }^{1}$, Tatiana Otero Mendelez ${ }^{1}$, Gladistone Correia Messias ${ }^{1}$, Cristiane

Magalhães Oliveira de Carvalho ${ }^{1}$, Gilson Soares Feitosa ${ }^{1^{*}}$

${ }^{1}$ Serviço de Cardiologia do Hospital Santa Izabel; Salvador, Bahia, Brazil

Background. COVID-19 is associated with a prothrombotic state leading to adverse clinical outcomes. Whether therapeutic anticoagulation improves outcomes in patients hospitalised with COVID-19 is unknown. We aimed to compare the efficacy and safety of therapeutic versus prophylactic anticoagulation in this population.

Methods. We did a pragmatic, open-label (with blinded adjudication), multicentre, randomised, controlled trial, at 31 sites in Brazil. Patients (aged $\geq 18$ years) hospitalised with COVID-19 and elevated D-dimer concentration, and who had COVID-19 symptoms for up to 14 days before randomisation, were randomly assigned $(1: 1)$ to receive either therapeutic or prophylactic anticoagulation. Therapeutic anticoagulation was inhospital oral rivaroxaban $(20 \mathrm{mg}$ or $15 \mathrm{mg}$ daily) for stable patients, or initial subcutaneous enoxaparin $(1 \mathrm{mg} / \mathrm{kg}$ twice per day) or intravenous unfractionated heparin (to achieve a $0 \cdot 3-0 \cdot 7 \mathrm{IU} / \mathrm{mL}$ anti-Xa concentration) for clinically unstable patients, followed by rivaroxaban to day 30 . Prophylactic anticoagulation was standard in-hospital enoxaparin or unfractionated heparin. The primary efficacy outcome was a hierarchical analysis of time to death, duration of hospitalisation, or duration of supplemental oxygen to day 30 , analysed with the win ratio method (a ratio $>1$ reflects a better outcome in the therapeutic anticoagulation group) in the intention-to-treat population. The primary safety outcome was major or clinically relevant non-major bleeding through 30 days. This study is registered with ClinicalTrials.gov (NCT04394377) and is completed.

Resumo de Artigo: Renato D. Lopes, Pedro Gabriel Melo de Barros e Silva, Remo H.M. Furtado et al. Therapeutic versus prophylactic anticoagulation for patients admitted to hospital with COVID-19 and elevated D-dimer concentration (ACTION): an openlabel, multicentre, randomised, controlled trial. Lancet 2021; 397: 2253-63. https://doi. org/10.1016/S0140-6736(21)01203-4. 
Findings. From June 24, 2020, to Feb 26, 2021, 3331 patients were screened and 615 were randomly allocated $(311[50 \%]$ to the therapeutic anticoagulation group and $304[50 \%]$ to the prophylactic anticoagulation group). $576(94 \%)$ were clinically stable and $39(6 \%)$ clinically unstable. One patient, in the therapeutic group, was lost to follow-up because of withdrawal of consent and was not included in the primary analysis. The primary efficacy outcome was not different between patients assigned therapeutic or prophylactic anticoagulation, with $28.899(34.8 \%)$ wins in the therapeutic group and $34.288(41.3 \%)$ in the prophylactic group (win ratio 0.86 [95\% CI 0.59-1.22], $p=0 \cdot 40$ ). Consistent results were seen in clinically stable and clinically unstable patients. The primary safety outcome of major or clinically relevant non-major bleeding occurred in $26(8 \%)$ patients assigned therapeutic anticoagulation and seven $(2 \%)$ assigned prophylactic anticoagulation (relative risk 3.64 [95\% CI 1.61-8.27], $\mathrm{p}=\mathbf{0 . 0 0 1 0}$ ). Allergic reaction to the study medication occurred in two $(1 \%)$ patients in the therapeutic anticoagulation group and three $(1 \%)$ in the prophylactic anticoagulation group.

Interpretation. In patients hospitalised with COVID-19 and elevated D-dimer concentration, in-hospital therapeutic anticoagulation with rivaroxaban or enoxaparin followed by rivaroxaban to day 30 did not improve clinical outcomes and increased bleeding compared with prophylactic anticoagulation. Therefore, use of therapeutic-dose rivaroxaban, and other direct oral anticoagulants, should be avoided in these patients in the absence of an evidence-based indication for oral anticoagulation.

Contexto.A COVID-19 está associada a um estado pró-trombótico que conduz a resultados clínicos adversos. Não se sabe se a anticoagulação terapêutica melhora os resultados em pacientes hospitalizados com COVID-19. Nosso objetivo neste estudo foi comparar a eficácia e segurança da anticoagulação terapêutica versus profilática nesta população.

Métodos Fizemos um ensaio clínico pragmático, aberto (duplo cego), multicêntrico, randomizado e controlado, em 31 centros no Brasil. Pacientes (com idade $\geq 18$ anos) hospitalizados com COVID-19 e concentração elevada de D-dímero, e que apresentavam sintomas de COVID-19 por até 14 dias antes da randomização, foram designados aleatoriamente (1:1) para receber anticoagulação terapêutica ou profilática. A anticoagulação terapêutica foi a rivaroxabana oral intra-hospitalar $(20 \mathrm{mg}$ ou $15 \mathrm{mg}$ por dia) para pacientes estáveis, ou enoxaparina subcutânea inicial ( $1 \mathrm{mg} / \mathrm{kg}$ duas vezes por dia) ou heparina não fracionada intravenosa (para atingir 0,3-0,7 IU / mL de concentração de anti-Xa) para pacientes clinicamente instáveis, seguido de rivaroxabana até o dia 30. A anticoagulação profilática foi a enoxaparina hospitalar padrão ou heparina não fracionada. O resultado de eficácia primário foi uma análise hierárquica do tempo até a morte, duração da hospitalização ou duração do oxigênio suplementar até o dia 30, analisada com o método de razão de acertos (uma razão> 1 reflete um melhor resultado no grupo de anticoagulação terapêutica) na intenção para tratar a população. O resultado de segurança primário foi sangramento maior ou não importante clinicamente relevante ao longo de 30 dias. Este estudo está registrado com ClinicalTrials.gov (NCT04394377) e está concluído.

Resultados. De 24 de junho de 2020 a 26 de fevereiro de 2021, 3.331 pacientes foram selecionados e 615 foram alocados aleatoriamente (311 [50\%] para o grupo de anticoagulação terapêutica e 304 [50\%] para o grupo de anticoagulação profilática).576 (94\%) eram clinicamente estáveis e 39 (6\%) clinicamente instáveis. Houve perda de seguimento em um paciente do grupo terapêutico, devido à retirada do consentimento e não foi incluído na análise primária. O resultado da eficácia primária não foi diferente entre os pacientes designados para anticoagulação terapêutica ou profilática, com $28,899(34,8 \%)$ no grupo terapêutico e $34,288(41,3 \%)$ no grupo profilático (proporção de acertos 0,86 [IC 95\% $0 \cdot 59-1 \cdot 22], \mathrm{p}=0 \cdot 40$ ). Resultados consistentes foram observados em pacientes clinicamente estáveis e clinicamente instáveis. O resultado de segurança primário para sangramento maior ou clinicamente relevante ocorreu em $26(8 \%)$ pacientes com tratamento terapêutico para anticoagulação e sete $(2 \%)$ para anticoagulação profilática (risco relativo $3 \cdot 64$ [IC 95\% $1 \cdot 61-8 \cdot 27], p=0,010)$. A reação alérgica ao medicamento do estudo ocorreu em dois (1\%) pacientes do grupo de anticoagulação terapêutica e três (1\%) do grupo de anticoagulação profilática.

Interpretação. Em pacientes hospitalizados com COVID-19 e concentração elevada de D-dímero, a terapêutica intra-hospitalar para anticoagulação, realizada com rivaroxabana ou enoxaparina, seguida de rivaroxabana até o dia 30, não melhorou os resultados clínicos e aumentou o sangramento em comparação com a anticoagulação profilática. Portanto, o uso de rivaroxabana em dose terapêutica e outros anticoagulantes orais diretos devem ser evitados nesses pacientes na ausência de uma indicação baseada em evidências para anticoagulação oral. 


\section{As Incertezas da Pandemia COVID-19}

A pandemia COVID-19 afetou drasticamente a vida humana em todo o planeta. Causada pelo vírus SARS-CoV-2, inicialmente identificado em Wuhan, na China, que rapidamente espalhouse por todo o mundo, foi declarada oficialmente como uma pandemia global pela Organização Mundial de Saúde no dia 11 de março de 2020. A severidade das manifestações da COVID-19 é bastante variável, desde uma maioria de pacientes assintomáticos até manifestações principalmente respiratórias que podem levar à insuficiência respiratóriaagudae aoóbito.Asmanifestaçõesmais graves estão comumente associadas a pacientes mais idosos e/ou com importantes comorbidades, embora, por motivos ainda desconhecidos, pode eventualmente afetar gravemente pessoas nãoidosas sem comorbidades.

Desde o início da pandemia COVID-19, inúmeras tentativas de tratamento para esta doença têm sido procuradas no melhor intuito de ajudar os pacientes e, dada a urgência da situação, encontrar uma solução rápida, mas raras foram testadas adequadamente. Muitas destas tentativas partem de uma ponderação em que, de um lado, há um suposto benefício a partir de observações ou frágeis evidências, confrontados contra o conhecido histórico de mínimos riscos. Sabe-se que experiências pessoais não sistematicamente controladas são sujeitas a um enorme número de vieses. Por uma série de motivos, o diálogo muitas vezes tem sido prejudicado e não há uma aceitação das incertezas como deveria ser a verdadeira ciência, atualmente tão evocada para encerrar precocemente debates.

Entre os vários pontos de incertezas, muitos alertas convergem a um risco trombótico elevado em pacientes com COVID-19, provavelmente maior que em outras doenças virais respiratórias, levando potencialmente a desfechos adversos graves. Desde então, médicos pelo mundo inteiro divergiam quanto ao benefício versus risco da anticoagulação plena destes pacientes, especialmente quando já apresentavam D-dímero elevado. Para dirimir esta dúvida, foi rapidamente planejado e executado o estudo ACTION, um dos estudos do grupo COALIZÃO.

\section{Desenolvimento do Estudo ACTION}

O estudo ACTION foi um estudo aberto com adjudicação cega, pragmático, randomizado, controlado, desenvolvido em 31 hospitais selecionados do Brasil. Como critérios de inclusão, eram aceitos pacientes internados com diagnóstico de COVID-19 com sintomas há menos de 14 dias, necessariamente com D-dímero elevado. Não poderiam participar do estudo pacientes com indicação formal de anticoagulação (por exemplo: fibrilação atrial, trombose venosa diagnosticada, dentre outras patologias), pacientes com clearance de creatinina inferior a $30 \mathrm{~mL} / \mathrm{min}$, plaquetopenia inferior a 50.000/mm3, uso de P2Y12 ou Aspirina em dose maior que $100 \mathrm{mg} /$ dia ou que tivessem, por algum outro motivo, alto risco de sangramento). Todos os pacientes eram informados e consentiram na participação deste ensaio clínico, que consistia em randomizar, na proporção 1:1, o tratamento com anticoagulação plena ou profilática usual.

A anticoagulação plena geralmente consistia no uso de Rivaroxabana $20 \mathrm{mg}$ ou $15 \mathrm{mg}$ /dia, por via oral, para os pacientes estáveis, ou heparina dose plena para os pacientes instáveis. $\mathrm{O}$ intuito era de manter anticoagulação plena por 30 dias, substituindo a via parenteral para via oral assim que possível. Já a anticoagulação profilática geralmente foi feita com heparina enquanto durasse a internação, conforme protocolo de cada hospital.

O desfecho primário de eficácia estabelecido foi uma análise hierárquica de tempo até a morte, tempo de internação e tempo de necessidade de suplementação de oxigênio, analisados até o $30^{\circ}$ dia de seguimento, através do método Win Ratio (Razão de Acertos), com base no princípio de intenção de tratar. $\mathrm{O}$ desfecho primário de segurança foi sangramento maior ou clinicamente relevante até o $30^{\circ}$ dia de seguimento. 
Assim, em um curto período de junho de 2020 a fevereiro de 2021, 615 pacientes foram randomizados e acompanhados nos centros selecionados. A grande maioria deles estava estável (94\%) no momento da randomização, a idade média em torno de 56 anos de idade, com discreto predomínio do sexo masculino. A randomização permitiu adequada distribuição de fatores de risco entre os grupos. O desfecho primário de eficácia não foi diferente entre os 2 grupos, com 28.899 (34,8\%) acertos do grupo terapêutico contra $34.288(41,3 \%)$ acertos do grupo profilático, com razão de acertos de 0,86 , com $\mathrm{p}=0,40$. Este resultado foi consistente em todas as análises de subgrupos pré-especificadas. Já a análise do desfecho primário de segurança mostrou resultado desfavorável ao grupo terapêutico, com 26 (8\%) pacientes apresentando sangramentos maiores ou clinicamente relevantes, contra $7(2 \%)$ pacientes do grupo profilático, atingindo $\mathrm{p}=0,001$ (Figura 1 e Tabela 1 / Figure 1, Table 1).

\section{O Método Win Ratio}

Um dos destaques do estudo ACTION foi a abordagem estatística do desfecho primário com uma metodologia à qual não estamos habituados. A forma convencional de reportar desfechos compostos em ensaios clínicos tem uma limitação de reunir eventos de magnitudes diferentes como desfecho único, sem hierarquia entre eles, frequentemente supervalorizando algum desfecho menos relevante. O método Win Ratio (Razão de Acertos) permite hierarquizar melhor os desfechos e possivelmente será mais frequente em futuros ensaios clínicos. Este método tem por base o confronto de todos os indivíduos de um grupo contra todos do outro, individualmente, com critérios pré-especificados para estabelecer o que seria o acerto de cada um ou o empate.

Assim, estabelece-se uma razão entre o número total de acertos da hipótese testada contra o número total de derrotas. Uma razão superior a 1 indica benefício, e inferior a 1 indica malefício. A relevância estatística é determinada pelo cálculo de intervalo de confiança de $95 \%$ e valor de $\mathrm{p}$.

No presente estudoACTION, foi feitaanálise hierarquizada, não pareada, estratificada conforme a condição estável ou instável na randomização. Primeiro, realizavam-se "confrontos" quanto à mortalidade em 30 dias, considerando empate caso nenhum dos dois comparados fosse a óbito ou caso a diferença no momento do óbito fosse de não mais de 1 dia.

Em seguida, havendo um empate, era comparado o tempo de internação hospitalar, considerando-se empate caso a diferença de duração de internação hospitalar fosse não mais que 2 dias.

Por fim, persistindo um empate, era avaliado o tempo de necessidade de oxigenioterapia, considerando-se empate caso a diferença de duração de uso fosse não superior a 2 dias entre as comparações.

\section{Interpretação e Aplicação Clínica}

O estudo ACTION traz importantes resultados. Pudemos aprender a partir deste estudo que, em pacientes internados por COVID-19 com D-dímero elevado, a anticoagulação terapêutica não trouxe benefícios clínicos significativos e ainda aumentou o risco de sangramentos maiores ou clinicamente significantes, quando comparado com anticoagulação profilática.

É natural procurarmos se algum subgrupo específico poderia favorecer-se do tratamento com anticoagulação plena, como pacientes com D-dímero mais elevado ou pacientes obesos ou pacientes mais graves. No entanto, todas as análises pré-especificadas de subgrupo não apontaram para um possível benefício da anticoagulação plena.

No trajeto da construção deste ensaio clínico, alguns pesquisadores encontraram dificuldades porque alguns médicos-assistentes não gostaram 
Figure 1. Primary outcome analysis

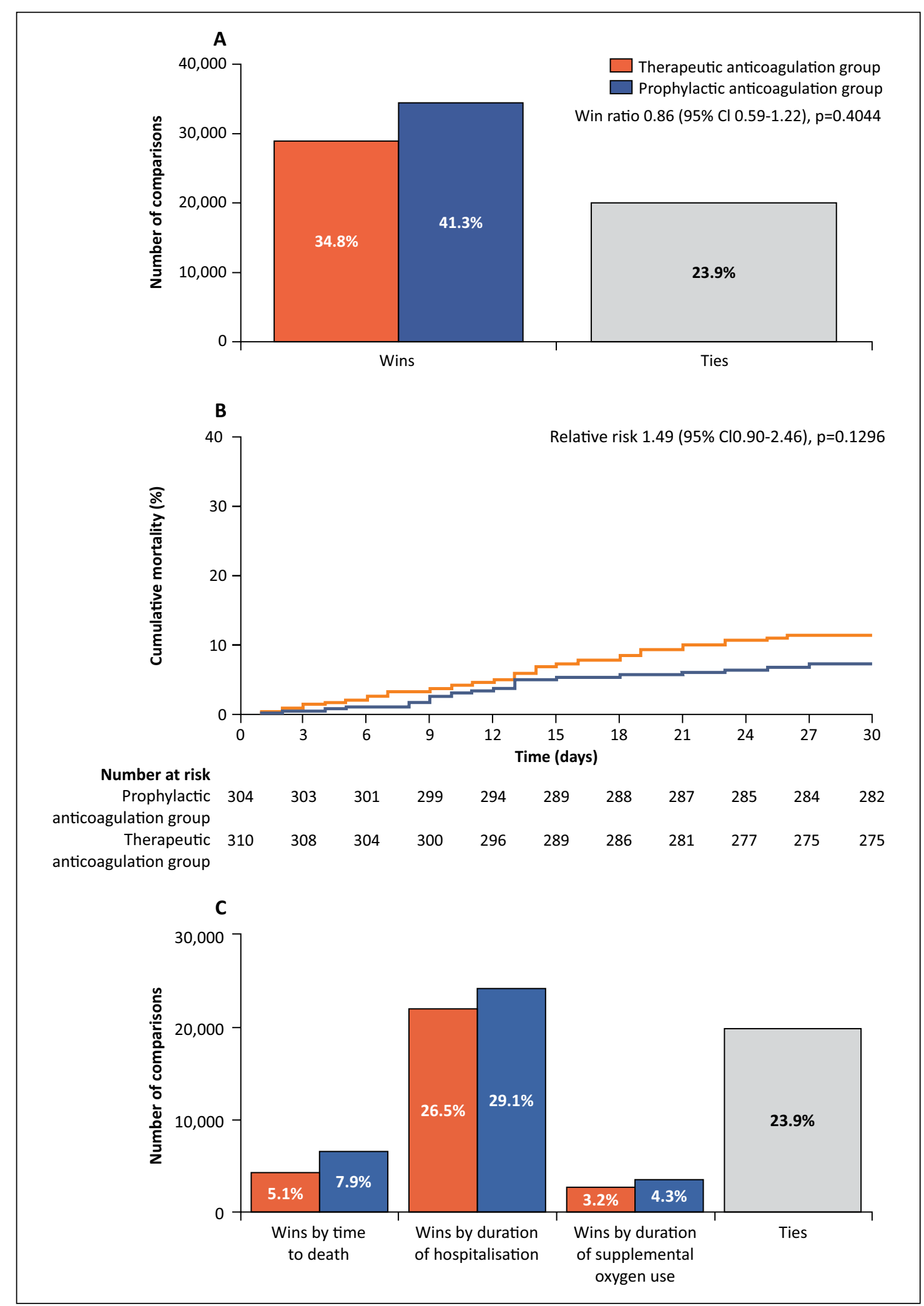

(A) Hierarchical win ratio analysis of death, duration of hospitalisation, and duration of oxygen use (primary outcome) through 30 days. (B) Cumulative mortality curves through 30 days in the two sudy groups. (C) Number of wins by group for individual components of the primary outcome. 
Table 1: 30-day efficacy and safety outcomes.

\begin{tabular}{|c|c|c|c|c|}
\hline & $\begin{array}{l}\text { Therapeutic anticoagulation } \\
\text { group }(n=310)\end{array}$ & $\begin{array}{l}\text { Prophylactic anticoagulation } \\
\text { group }(n=304)\end{array}$ & Effect $(95 \% \mathrm{Cl})$ & p value \\
\hline \multicolumn{5}{|l|}{ Efficacy outcomes } \\
\hline Composite thrombotic outcome* & $23(7 \%)$ & $30(10 \%)$ & RR $0.75(0.45-1.26)$ & 0.32 \\
\hline Venous thromboembolism $†$ & $11(4 \%)$ & $18(6 \%)$ & RR0.60 (0.29-1.25) & 0.19 \\
\hline Deep vein thrombosis & $5(2 \%)$ & $5(2 \%)$ & RR $0.98(0.29-3.35)$ & 1.00 \\
\hline Pulmonary embolism & $7(2 \%)$ & $13(4 \%)$ & RR0.53 (0.21-1.31) & 0.18 \\
\hline Myocardial infarction & $13(4 \%)$ & $14(5 \%)$ & RR $0.91(0.44-1.91)$ & 0.85 \\
\hline Stroke & $1(<1 \%)$ & 0 & .. & .. \\
\hline Major adverse limb event & 0 & $1(<1 \%)$ &.. &.. \\
\hline \\
\hline Death & $35(11 \%)$ & $23(8 \%)$ & RR 1.49 (0.90-2.46) & 0.13 \\
\hline Cardiovascular & $6(2 \%)$ & 0 &.. &.. \\
\hline Non-cardiovascular & 29 9\%) & $22(7 \%)$ &.. & .. \\
\hline Unknown & 0 & $1(<1 \%)$ &.. &.. \\
\hline Rehospitalisation & $2(1 \%)$ & $5(2 \%)$ & RR $0.39(0.08-2.01)$ & 0.28 \\
\hline $\begin{array}{l}\text { World health Association 8-point ordinal } \\
\text { scale at end of } 30 \text { days }\end{array}$ & .. & .. & $\begin{array}{l}\text { Proportional OR } 1.35 \\
\qquad(0.85-2.16)\end{array}$ & 0.21 \\
\hline Out of hospital with no oxygen therapy & $263(85 \%)$ & $268(88 \%)$ & .. & .. \\
\hline Out of hospital with oxygen therapy & 0 & 0 & .. & .. \\
\hline Hospitalised with no oxygen therapy & $3(1 \%)$ & $5(2 \%)$ & .. & .. \\
\hline $\begin{array}{l}\text { Hospitalides with oxygen by mask or nasal } \\
\text { catheter }\end{array}$ & $8(3 \%)$ & $2(1 \%)$ & .. & .. \\
\hline $\begin{array}{l}\text { Hospitalised with non-invasive ventilation } \\
\text { or high flow oxygen }\end{array}$ & 0 & 0 & .. &.. \\
\hline $\begin{array}{l}\text { Hospitalised with invasive mechanical } \\
\text { ventilation without additional support }\end{array}$ & $1(<1 \%)$ & $4(1 \%)$ & .. & .. \\
\hline $\begin{array}{l}\text { Hospitalised with invasive mechanical } \\
\text { ventilation with additional organ support }\end{array}$ & 0 & $2(1 \%)$ & .. &.. \\
\hline Death & $35(11 \%)$ & $23(8 \%)$ & .. & .. \\
\hline \multicolumn{5}{|l|}{ Safety outcomes } \\
\hline $\begin{array}{l}\text { Major bleeding or clinically relevant } \\
\text { non-major bleeding (ISTH definitions) }\end{array}$ & $26(8 \%)$ & $7(2 \%)$ & RR $3.64(1.61-8.27)$ & 0.0010 \\
\hline Major bleeding & $10(3 \%)$ & $4(1 \%)$ & RR $2.45(0.78-7.73)$ & 0.18 \\
\hline Clinically relevant non-major bleeding & $16(5 \%)$ & $3(1 \%)$ & RR 5.23 (1.54-17.77) & 0.0039 \\
\hline Any bleeding & $36(12 \%)$ & $9(3 \%)$ & RR $3.92(1.92-8.00)$ & $<0.0001$ \\
\hline \multicolumn{5}{|l|}{ Combined efficacy and safety outcome } \\
\hline Net benefit ¥ & $56(18 \%)$ & $47(15 \%)$ & RR $1.17(0.82-1.66)$ & 0.45 \\
\hline
\end{tabular}

de anticoagular plenamente um paciente que não tinha critérios estabelecidos de anticoagulação e porque outros não concordavam com a ideia de não anticoagular um paciente COVID-19 com D-dímero elevado, mesmo sem evidência robusta. Esta dicotomia de condutas opostas para um mesmo cenário corrobora a necessidade de um bom ensaio clínico randomizado para nos guiar.

A partir deste estudo, temos um bom respaldo para não anticoagular plenamente um paciente com COVID-19, a menos que exista uma indicação específica, e a simples elevação do D-dímero não se trata de uma indicação formal. 